\title{
Effect of lactoferrin protein on red blood cells and macrophages: mechanism of parasite-host interaction
}

This article was published in the following Dove Press journal:

Drug Design, Development and Therapy

27 July 2015

Number of times this article has been viewed

\author{
Namrata Anand' \\ Rupinder K Kanwar ${ }^{2}$ \\ Mohan Lal Dubey' \\ R K Vahishta ${ }^{3}$ \\ Rakesh Sehgal',* \\ Anita K Verma ${ }^{4}$ \\ Jagat R Kanwar ${ }^{2, *}$
}

'Department of Medical Parasitology, Postgraduate Institute of Medical Education and Research, Chandigarh, India; ${ }^{2}$ Nanomedicine Laboratory of Immunology and Molecular Biomedical Research, School of Medicine, Molecular and Medical Research Strategic Research Centre, Faculty of Health, Deakin University, Geelong, VIC, Australia; ${ }^{3}$ Department of Histopathology, Postgraduate Institute of Medical Education and Research, Chandigarh, ${ }^{4}$ Nanobiotech Laboratory, Department of Zoology, Kirorimal College, University of Delhi, Delhi, India

*These authors contributed equally to this work

Correspondence: Rakesh Sehgal Department of Medical Parasitology, Postgraduate Institute of Medical Education and Research, Madhya Marg, Sector-12, Chandigarh, PIN-I600I2, India Email sehgalpgi@gmail.com

Jagat R Kanwar

Nanomedicine-Laboratory of Immunology and Molecular Biomedical Research, School of Medicine, Molecular and Medical Research Strategic Research Centre, Faculty of Health, Geelong Technology Precinct, Deakin University, Pigdons Road, Waurn Ponds, VIC 3216, Australia

Email jagat.kanwar@deakin.edu.au
Background: Lactoferrin is a natural multifunctional protein known to have antitumor, antimicrobial, and anti-inflammatory activity. Apart from its antimicrobial effects, lactoferrin is known to boost the immune response by enhancing antioxidants. Lactoferrin exists in various forms depending on its iron saturation. The present study was done to observe the effect of lactoferrin, isolated from bovine and buffalo colostrum, on red blood cells (RBCs) and macrophages (human monocytic cell line-derived macrophages THP1 cells).

Methods: Lactoferrin obtained from both species and in different iron saturation forms were used in the present study, and treatment of host cells were given with different forms of lactoferrin at different concentrations. These treated host cells were used for various studies, including morphometric analysis, viability by MTT assay, survivin gene expression, production of reactive oxygen species, phagocytic properties, invasion assay, and Toll-like receptor-4, Toll-like receptor-9, and MDR1 expression, to investigate the interaction between lactoferrin and host cells and the possible mechanism of action with regard to parasitic infections.

Results: The mechanism of interaction between host cells and lactoferrin have shown various aspects of gene expression and cellular activity depending on the degree of iron saturation of lactoferrin. A significant increase $(P<0.05)$ in production of reactive oxygen species, phagocytic activity, and Toll-like receptor expression was observed in host cells incubated with ironsaturated lactoferrin when compared with an untreated control group. However, there was no significant $(P>0.05)$ change in percentage viability in the different groups of host cells treated, and no downregulation of survivin gene expression was found at 48 hours post-incubation. Upregulation of the Toll-like receptor and downregulation of the P-gp gene confirmed the immunomodulatory potential of lactoferrin protein.

Conclusion: The present study details the interaction between lactoferrin and parasite host cells, ie, RBCs and macrophages, using various cellular processes and expression studies. The study reveals the possible mechanism of action against various intracellular pathogens such as Toxoplasma, Plasmodium, Leishmania, Trypanosoma, and Mycobacterium. The presence of iron in lactoferrin plays an important role in enhancing the various activities taking place inside these cells. This work provides a lot of information about targeting lactoferrin against many parasitic infections which can rule out the exact pathways for inhibition of diseases caused by intracellular microbes mainly targeting RBCs and macrophages for their survival. Therefore, this initial study can serve as a baseline for further evaluation of the mechanism of action of lactoferrin against parasitic diseases, which is not fully understood to date.

Keywords: lactoferrin, phagocytosis, cytotoxicity, morphometric analysis

\section{Introduction}

Lactoferrin is a multifunctional glycoprotein belonging to the transferrin family. These proteins are capable of transferring and accepting iron ions from the surrounding 
environment depending on the iron content of the protein. ${ }^{1}$ There are three forms of lactoferrin, according to its iron saturation: apolactoferrin (iron-free), a monoferric form (containing one ferric ion), and hololactoferrin (containing two iron ions). ${ }^{2}$ Lactoferrin was first isolated by Sorensen and Sorensen from bovine milk in $1939 .{ }^{3}$ The protein is naturally present in large amounts in colostrum and milk. Lactoferrin performs many biological functions, and has antimicrobial, antitumor, antioxidant, antitumor, and immunomodulatory activity. ${ }^{4}$ The ability to keep iron bound even at low pH is important, especially at sites of infection and inflammation where, due to the metabolic activity of bacteria, the $\mathrm{pH}$ may fall under 4.5. In such a situation, lactoferrin also binds iron released from transferrin, which prevents its further use for bacterial proliferation. ${ }^{5}$ Lactoferrin is known to inhibit the growth of various tumor cell lines through production of oxidative stress, such as in the case of leukemia cell lines. ${ }^{6}$

Earlier studies have shown the effect of lactoferrin on macrophages, blood cells, and tumor cell lines. This protein has been known to kill tumors when given orally to mice. ${ }^{7}$ There has been very little research on the inhibitory effect of lactoferrin protein against parasitic diseases, for which we choose the most popular host cells for parasitic infections. Red blood cells (RBCs) are host cells for Plasmodium and Babesia, and macrophages have been targeted by Leishmania, Toxoplasma, and Mycobacterium. Previous studies have shown that lactoferrin protects human RBCs from oxidative stress in its monoferric form, and macrophages show increased levels of reactive oxygen species (ROS) after treatment with bovine lactoferrin. ${ }^{8}$ These studies have yielded interesting results and created enthusiasm for further indepth study of the various biological reactions taking place inside lactoferrin-treated cells as well as normal cells. Therefore, the present study was undertaken in an attempt to understand the mechanism of interaction between lactoferrin and host cells by studying various parameters on human RBCs and macrophages, treated as host cells. Treatment of host cells with lactoferrin and its interaction with parasitic infections has been detailed in the present paper.

Survivin is a gene that is expressed only in live tumor cells and was included in the present study through analysis of various gene expression and metabolic processes to check the survival of THP1 cells after lactoferrin treatment. Survivin expression has been studied in tumor cells treated with higher doses of lactoferrin and was found to be downregulated due to treatment of tumor. ${ }^{9}$
The immunomodulatory and anti-inflammatory activity of lactoferrin protein has been shown to occur via signaling pathways including various Toll-like receptors (TLRs). ${ }^{10,11}$ TLR-2, TLR-4, and TLR-9 are activated in many infections involving virus, parasites, and bacteria. Lactoferrin has been shown to reduce the activity of Epstein Bar virus ${ }^{12}$ via interaction with its coreceptor. It is also known to activate macrophages through TLR-4, involving direct and indirect signaling pathways. ${ }^{13}$ TLR signaling in macrophages that have lactoferrin on their surface can activate different TLRs against various parasitic infections, so it is necessary to study the expression of different TLRs after stimulation of the host cell with lactoferrin.

MDR1 is used to investigate the expression of P-gp, a resistance marker gene in tumor cells. As there are a lot of problems related to antibiotic drug resistance in infectious diseases, we wanted to check the expression of this gene in the presence of lactoferrin. Therefore, it is important to determine whether it is helpful in normal conditions and also if it is helpful in resistant tumor cells whether to enhance the resistance or down regulate it.

Lactoferrin protein has been isolated from five types of mammals, namely, human, cow, buffalo, goat and mare, and comparative studies have been done to differentiate between the various origins. ${ }^{14}$ The main difference lies in the structural domain, which is different from one species to another. Many studies have used the bovine and human lactoferrins, but no studies have been done using buffalo lactoferrin. This is the first study investigating the interaction between buffalo lactoferrin and host cells. How we can target this protein in parasitic diseases by studying the basic mechanism of action, and whether it differs from human lactoferrin and bovine lactoferrin has been tried to determine in the present study.

\section{Materials and methods}

The bovine lactoferrin used in this study was obtained from Jagat Kanwar, Deakin University, Australia, and the buffalo lactoferrin was purified from buffalo colostrum using an ion exchange chromatography method described elsewhere. ${ }^{15}$

Three types of lactoferrin proteins of bovine and buffalo origin were prepared on the basis of their different iron saturations. Iron-saturated lactoferrin was prepared according to a method previously described by Bates et al. ${ }^{16}$ Iron estimation for all forms of lactoferrin was done using a previously described method with minor modifications. ${ }^{17}$ 
Apolactoferrin was prepared using the method devised by Feng et al with minor modifications, ${ }^{18}$ and was found to be $8 \%-10 \%$ saturated with iron; the monoferric form was found to be $40 \%-65 \%$ saturated and the holo form was found to be $85 \%-95 \%$ saturated.

\section{Host RBCs and macrophages}

The RBCs were obtained from a healthy human donor (Rotary and Blood Bank Society, Sec-37C Chandigarh, India). Use of human RBCs was approved by the institutional ethics committee, Postgraduate Institute of Medical Education and Research, Chandigarh, India. The THP1 cell line was purchased from National Cell Culture Science, Pune, India. Cell line was maintained in culture medium (Roswell Park Memorial Institute [RPMI] 1640 and 10\% fetal bovine serum supplemented with L-glutamine, $25 \mathrm{mM}$ HEPES buffer, and penicillin-streptomycin) at $37^{\circ} \mathrm{C}$ with $5 \% \mathrm{CO}_{2}$ and $90 \%$ humidity. The THP1 cells were differentiated to macrophages with the assistance of phormol myristate- 13 acetate at a final concentration of $10 \mathrm{nM}$ for 48 hours.

\section{Preparation of RBCs}

Fresh blood is collected from donor and collected in an anticoagulant blood collection bag. The whole blood was dispensed in aliquots into glass bottles. A small quantity of blood from each glass bottle was transferred into centrifuge tubes and spun at 1,500 rpm for 10 minutes. The plasma and buffy coat were removed aseptically. The remaining white blood cells and platelets were removed by washing with medium twice. The final blood volume was mixed with growth medium containing AlbuMAX ${ }^{\circledR}$ and stored at $4^{\circ} \mathrm{C}$ for further use.

\section{Treatment of RBCs and macrophages with lactoferrin}

Differentiated THP1 cells and fresh RBCs at a concentration of $1 \times 10^{6} / \mathrm{mL}$ were incubated with lactoferrin in 24-well tissue culture plates and 96-well plates, respectively. Concentrations ranging from 10, 20, 40 and $50 \mu \mathrm{g} / \mathrm{mL}$ were used, and cells were incubated for 24 and 48 hours for initial dose standardization. Optimum concentrations were selected and RBCs and macrophages were subjected to various parameters, ie, morphology by Giemsa staining, production of ROS, capacity of phagocytosis, and survivin gene expression by real-time polymerase chain reaction. Expression studies of TLR and drug resistance by MDR1 were studied for better understanding the mechanism of action of lactoferrin.

\section{Morphometric analysis by Giemsa staining}

After 48 hours of incubation, the macrophages and RBCs were fixed with 100\% methanol and stained with Giemsa for 30 minutes, and then observed by morphometric analysis.

\section{Production of reactive oxygen species}

The standard protocol was followed, with slight modifications. ${ }^{19}$ The treated macrophages and RBCs were investigated for production of ROS, detected by 2-7, dichlorofluorescein (DCFH) dye used at a final concentration of $5 \mu \mathrm{M}$ for all experiments. The cells were incubated with the dye for 15 minutes at $37^{\circ} \mathrm{C}$, washed twice to remove the extra dye, and then fixed with $4 \%$ paraformaldehyde for 1 hour at $4^{\circ} \mathrm{C}$. The cells were washed twice with phosphate-buffered saline (PBS) and acquired by flow cytometery analysis using FACS Caliber. Fluorescein isothiocyanate laser at $485 \mathrm{~nm}$ was acquired for ROS dye. Before starting the experiment, cytometry setup tracking was run, and the voltage was set up accordingly. Macrophages were acquired in a forwardscattered light plot, fluorescence was measured in each experiment, and was calculated in all experiments.

\section{Viability of macrophages by MTT assay}

The treated macrophages were incubated with MTT [3-(4,5dimethylthiazol-2-yl)-2,5-diphenyltetrazolium bromide] solution $(5 \mathrm{mg} / \mathrm{mL})$ at 44 hours of incubation. After 48 hours of incubation, all medium was removed from the wells and $50-100 \mu \mathrm{L}$ of dimethyl sulfoxide was added to each well. The violet crystals were dissolved by keeping the plates at $37^{\circ} \mathrm{C}$ for $15-20$ minutes, and reading was taken at $595 \mathrm{~nm}$. Percent viability was calculated following a standard protocol described elsewhere. ${ }^{20}$

\section{Measurement of phagocytic capacity}

Treated and untreated macrophages were incubated with latex beads to investigate their phagocytic properties. Phagocytosis is the process by which macrophages engulf intracellular microbes/particles. How different forms of Lactoferrin show different degree of phagocytosis has been studied in this section.

The macrophages were incubated with $1 \mu \mathrm{m}$ fluorescent latex beads (Sigma, St Louis, MO, USA) at a concentration of $5 \times 10^{8}$ beads $/ \mathrm{mL}$ for 15 minutes at $37^{\circ} \mathrm{C}$. Extra nonengulfed 
beads were removed by two washes in PBS, and the cells were fixed with paraformaldehyde and observed by light microscopy. The intensity of fluorescence of the engulfed macrophages was also measured by flow cytometry to confirm the light microscopy results. The mean fluorescence intensity of the targeted macrophages was further confirmed with confocal images.

\section{Survivin, MDRI, and TLR gene expression}

The effect of lactoferrin on macrophages was investigated by studying the expression of various genes using real-time polymerase chain reaction (LC 480 Roche, Basel, Switzerland). The cells were incubated with different types of lactoferrin at optimum dose for 48 hours. After incubation, the cells were detached from the plates using Accutase enzyme solution. Total RNA was extracted using TRIzol reagent and complementary DNA was prepared (Thermo Fisher cDNA kit, Loughborough, UK). Complementary DNA was amplified for the survivin, ${ }^{21}$ MDR 1, ${ }^{22}$ TLR 4, and TLR 9 genes to check the fold increase or decrease in expression relative to glyceraldehyde-3-phosphate dehydrogenase (GAPDH), ${ }^{23}$ the housekeeping gene. The TLR inhibitor OxPAPC was used to inhibit TLR signaling for 24 hours. Expression studies was then conducted using the method described earlier to demonstrate the effect of lactoferrin. Primer sequences with the annealing temperatures are given in Table 1.

\section{Assay for intracellular invasion by Toxoplasma gondii}

After treatment with the different lactoferrins, macrophages were infected with Toxoplasma gondii tachyzoites at a ratio of 1:5 (one macrophage and 5 tachyzoites), and after 5 hours, the number of intracellular tachyzoites were used to calculate the mean number of invasive events.

Table I Primer sequences and annealing temperature for different genes

\begin{tabular}{|c|c|c|}
\hline Gene & Primer sequence & $\begin{array}{l}\text { Annealing } \\
\text { temperature }\end{array}$ \\
\hline Survivin forward & GCCCAGTGTTTCTTCTGCTT & $60^{\circ} \mathrm{C}$ \\
\hline Survivin reverse & CC GGACGAATGCTTTTTATG & \\
\hline TLR-4 forward & CCAGTGAGGATGATGCCAGAAT & $55^{\circ} \mathrm{C}$ \\
\hline TLR-4 reverse & GCCATGGCTGGGATCAGAGT & \\
\hline TLR-9 forward & GGACCTCTGGTACTGCTTCCA & $55^{\circ} \mathrm{C}$ \\
\hline TLR-9 reverse & AAGCTCGTTGTACACCCAGTCT & \\
\hline MDRI forward & GCTGG TGCTGCTTACATTC & $55^{\circ} \mathrm{C}$ \\
\hline MDRI reverse & GCTGACAGTCCAAGAACAGG & \\
\hline GAPDH forward & AGCCACATCGCTCAGACAC & $56^{\circ} \mathrm{C}$ \\
\hline GAPDH reverse & GCCCAATACGACCAAATCC & \\
\hline
\end{tabular}

Abbreviations: GADPH, glyceraldehyde-3-phosphate dehydrogenase; TLR, Tolllike receptor.

\section{Statistical analysis}

The size variation, phagocytic capacity, ROS production, and number of invasive parasites between different treatment groups were compared with the untreated group; for expression studies the difference between each group was determined by Student's $t$-test and between the groups in case of MTT assay by one-way analysis of variance test. Differences between groups were accepted as being statistically significant at $p<0.05, p<0.05$, and $p<0.005$. All results are average of three experiments when performed in duplicates.

\section{Results}

\section{No morphometric changes in RBCs after treatment with lactoferrin}

After treatment of the human RBCs with the different types of lactoferrin, no color was observed in cultured supernatants, indicating no hemolysis of RBCs. No significant difference in the morphology of RBCs was observed on Giemsa staining at concentrations of $10-40 \mu \mathrm{g} / \mathrm{mL}$ on comparison with the untreated control group (Figure 1A). The results of morphological analysis by Giemsa staining were analyzed, and $40 \mu \mathrm{g} / \mathrm{mL}$ concentration of protein was chosen and used for further experiments, such as measurement of ROS production (Figure 1B).

\section{Iron-saturated lactoferrin showed increased ROS production in RBCs}

Lactoferrin showed different amounts of ROS production depending on its degree of iron saturation. When treated with lactoferrins at a concentration of $40 \mu \mathrm{g} / \mathrm{mL}$, RBCs showed a different pattern of ROS production. The apolactoferrin form used at this concentration showed significantly decreased levels of ROS production compared with the untreated control group, whereas iron-saturated bovine lactoferrin showed a significant 2-fold increase in ROS level compared with the untreated group at 48 hours post-incubation (Figure 2).

\section{THPI macrophage cells survived} in a dose-/time-dependent manner

The viability of the treated macrophages were observed using MTT viability assay. There was no significant difference observed in percent cell viability when compared with untreated macrophages at 10,20,40 and $50 \mu \mathrm{g} / \mathrm{mL} 48$ hours posttreatment (Figure 3A). Morphological changes in the macrophages, including size and diameter, were observed 

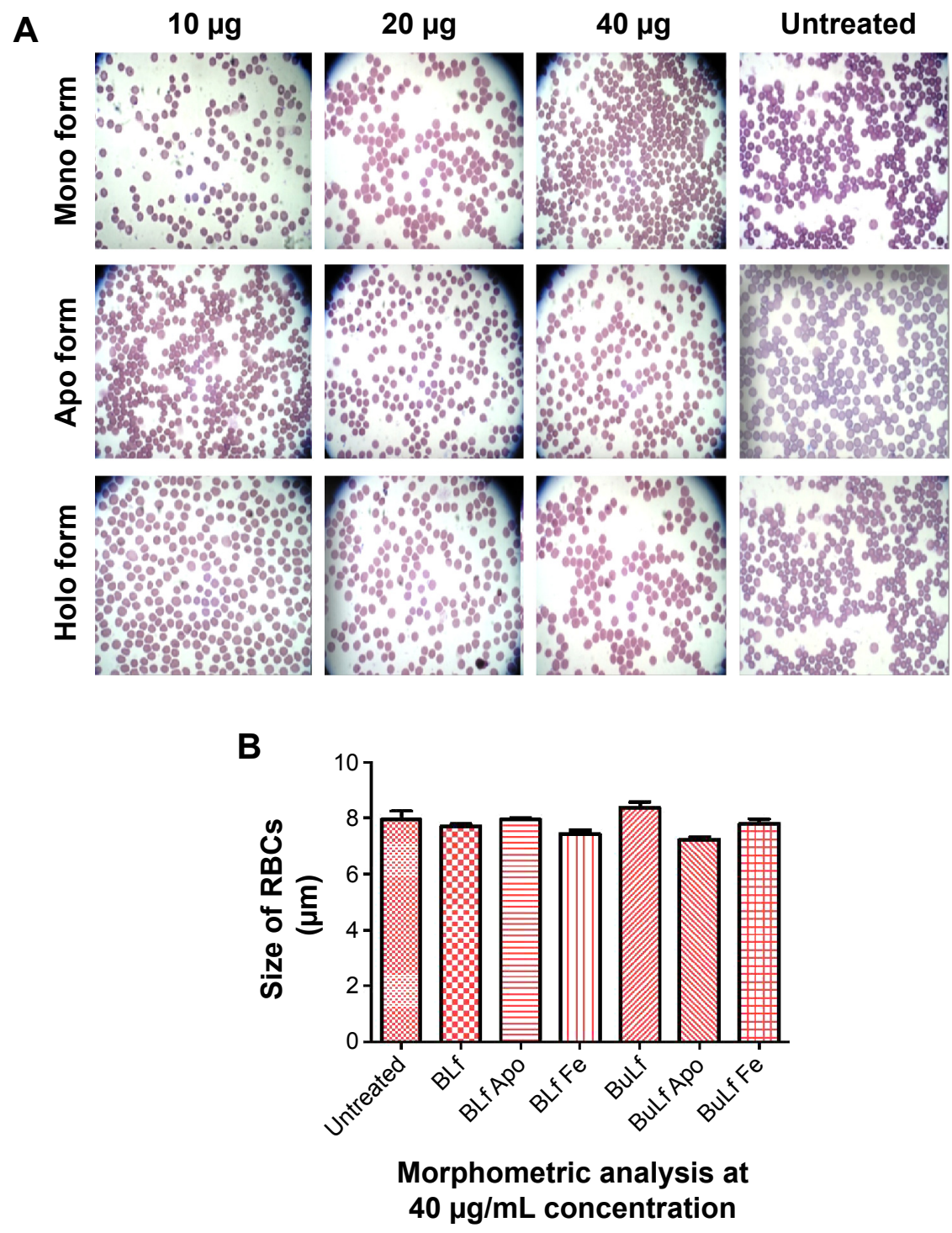

Figure I (A) Giemsa stain microscopy of red blood cells treated with different forms of lactoferrin at different concentrations (I00x) and the untreated group. This experiment was repeated in triplicate. Morphological characteristics, ie, shape, size, and diameter, were the same in the different treated red blood cells compared with the untreated group. (B) Morphometric analysis of RBCs treated for 48 hours with the different proteins at a concentration of $40 \mu \mathrm{g} / \mathrm{mL}$. No significant difference was found between the treated and untreated groups.

Abbreviations: BLf, bovine lactoferrin; BLf Fe, bovine apolactoferrin; BLf Fe, iron-saturated bovine lactoferrin; BuLf, buffalo lactoferrin; BuLf Apo, buffalo apolactoferrin; BuLf Fe, iron-saturated buffalo lactoferrin; RBSs, red blood cells.

after treatment with the lactoferrins, and no significant difference in size was found between the treated and untreated macrophages (Figure 3B and C).

\section{Iron-saturated lactoferrin enhanced ROS production, phagocytic capacity, and expression of TLR and survivin in macrophages}

When concentrations ranging from 10 to $40 \mu \mathrm{g} / \mathrm{mL}$ were used from all types of proteins, there was a significant increase in ROS production from a lower to a higher dose, with a 2-4-fold increase in intensity (Figure 4A and B). Iron-saturated lactoferrin protein has been shown to generate many free radical ions which can be helpful for inhibiting a variety of tumors, intracellular parasites, and microbes. After investigation of these parameters, the dose was standardized and the $20 \mu \mathrm{g} / \mathrm{mL}$ concentration was chosen to evaluate further parameters on the macrophages.

After treatment with the different lactoferricin at a concentration of $20 \mu \mathrm{g} / \mathrm{mL}$, the phagocytic capacity of the macrophages was measured (Figure 5A). Interestingly there was a higher mean fluorescence intensity in ironsaturated lactoferrin-treated macrophages compared with 

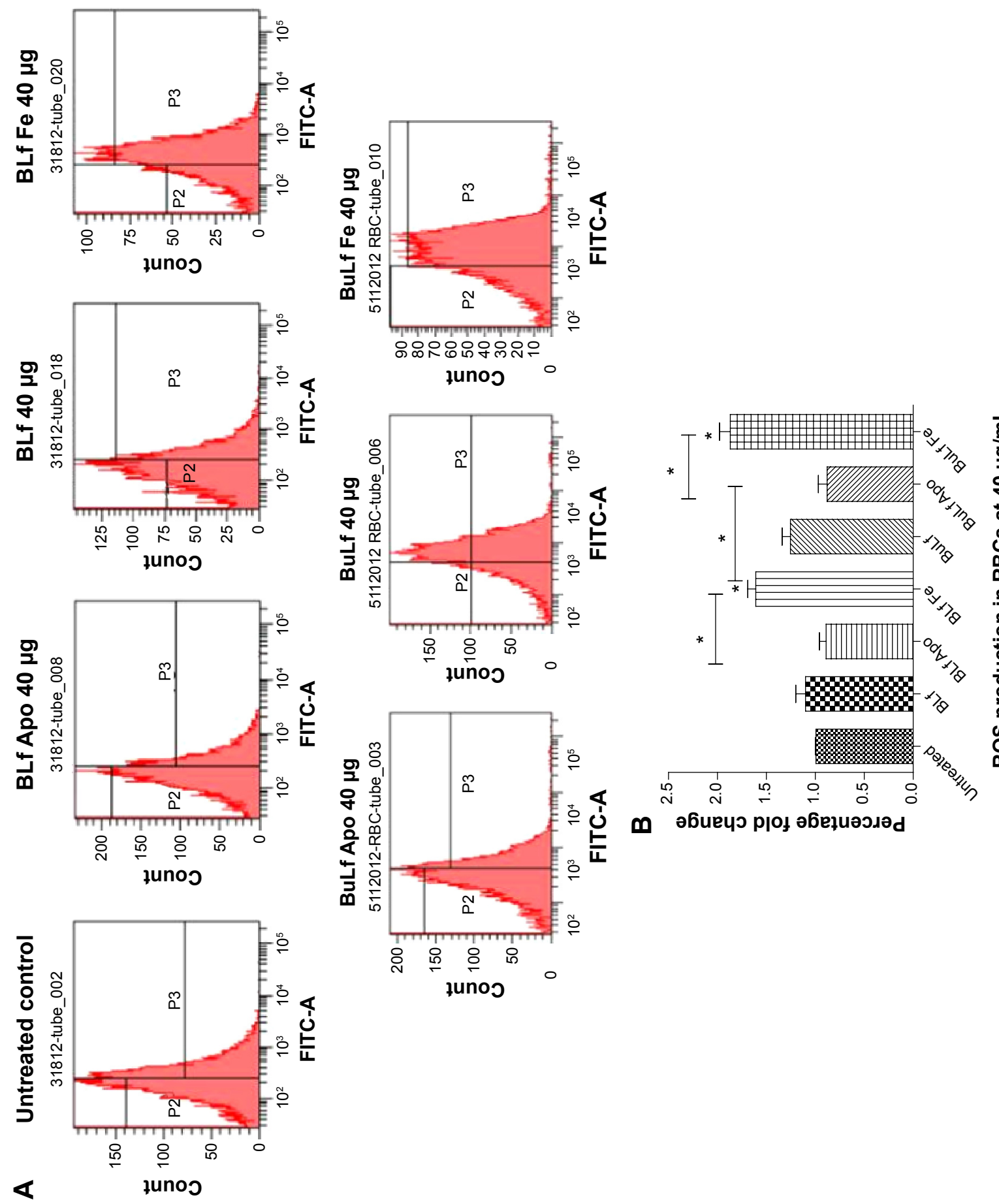
u sobeydosoew to әz!s
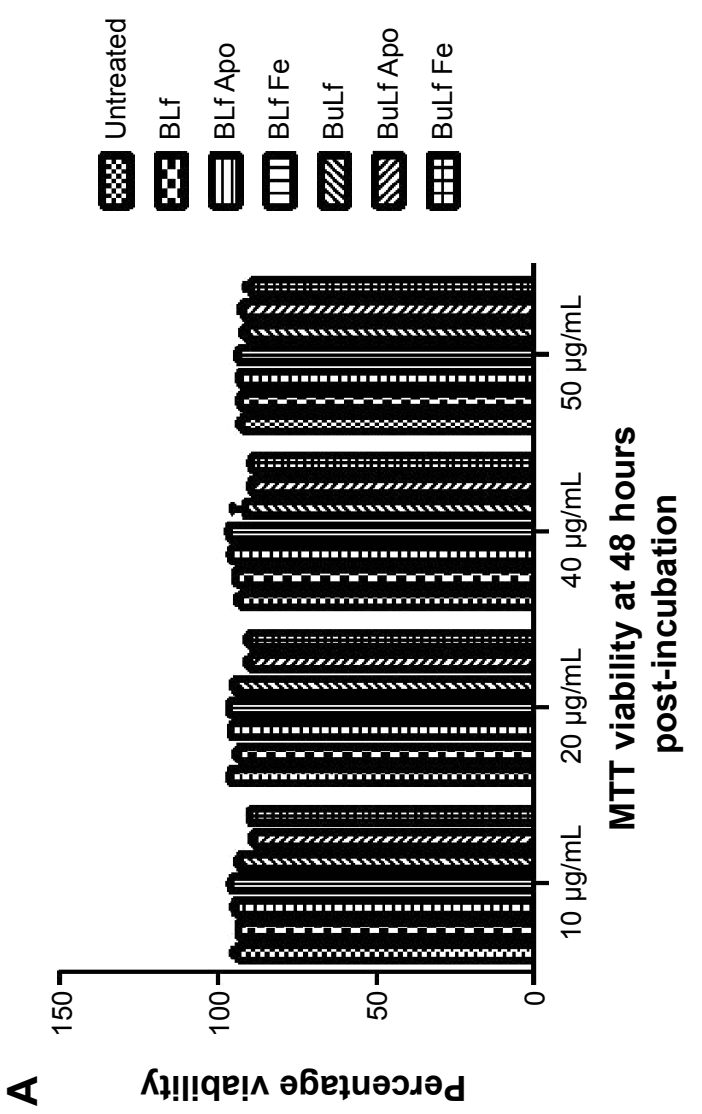

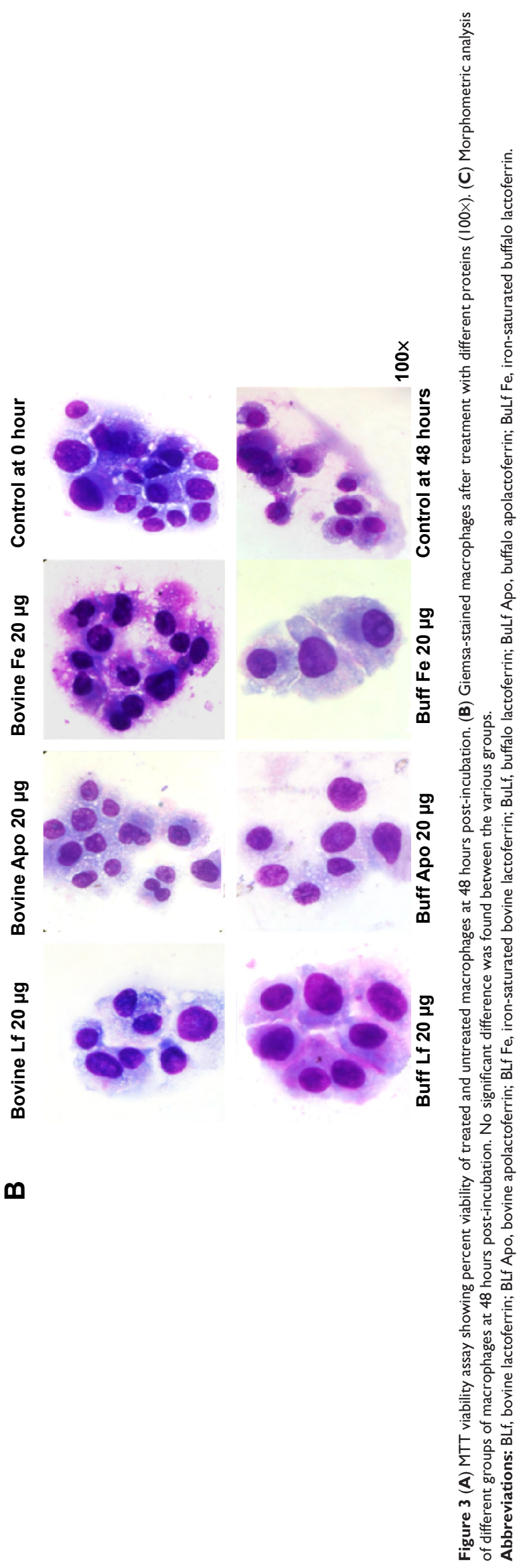





B



Figure 4 (A) Significant fold increase in ROS production at increasing dose concentrations ranging from 10 to $50 \mu \mathrm{g} / \mathrm{mL}$ of the various lactoferrins. (B) Significant $(P<0.005)$ fold increase in ROS production when used at $20 \mu \mathrm{g} / \mathrm{mL}$ compared with the untreated group and within the group $(* P<0.05)$ shown by bar diagram. Each value is the replicate of three experimental values. $* P<0.05 ; * * P<0.005$.

Abbreviations: Lf, lactoferrin; ROS, reactive oxygen species; BLf, bovine lactoferrin; BLf Apo, bovine apolactoferrin; BLf Fe, iron-saturated bovine lactoferrin; BuLf, buffalo lactoferrin; BuLf Apo, buffalo apolactoferrin; BuLf Fe, iron-saturated buffalo lactoferrin.

the apolactoferrin-treated and native lactoferrin-treated groups (Figure 5B). The fluorescence intensity results were confirmed by confocal microscopy, where greater numbers of beads were found to be engulfed by macrophages treated with iron-saturated lactoferrin compared with the untreated control and other lactoferrin groups. The phagocytic capacity of the macrophages was further confirmed by an invasion assay. The maximum number of infected macrophages and intracellular tachyzoites per macrophage were found in the iron-saturated lactoferrin-treated group and the minimum number were found in the apolactoferrin group (Figure 5C). The mean number of intracellular tachyzoites were found to be higher in the iron-saturated lactoferrin group when compared with the other groups (Figure 5D).

After treatment with the iron-saturated lactoferrins, higher expression of survivin, TLR-4, and TLR-9 with respect to the housekeeping gene, GAPDH gene, was observed at 48 hours post-incubation (Figure 6). After inhibition of TLRs, less expression of TLR-4 and TLR-9 was found, indicating that lactoferrin is essential for stimulation of the TLR signaling pathways to activate against microbes or immunomodulatory action (Figure 6). However, the real mechanism of action is not known and needs to be detailed out. These expressions showed function of lactoferrin protein in their activation, which will help in further recognition of pathogens. The signaling pathway has not been studied; however, our present results suggest that lactoferrin activates macrophages via TLR-4 signaling. ${ }^{24}$ Further, TLR-9 has been known to attach to the malarial parasite, and its activation by lactoferrin may help to destroy the parasite.

Whereas MDR1 showed slightly low expression when treated with iron-saturated lactoferrin compared with untreated group. Low expression may help in decreasing drug resistance of pathogens which express its alteration in normal function.

This can be a helpful criteria in drug resistance pathogens which will lead to a decrease in the resistance mechanism. This might be due to the inhibitory properties of iron-saturated lactoferrin and therefore will be helpful in retaining the drug inside the cell. The exact mechanism of downregulation of the MDR1 gene was not identified and needs to be investigated further.

It was concluded from the above results that macrophages become activated after treatment with iron-saturated lactoferrin and perform various metabolic activities leading to recognition and binding, engulfment, and inhibition of pathogens through phagocytosis. These cellular processes have clarified the mechanism of action of lactoferrin proteins with different degrees of iron saturation. Whereas, the above activation will not be observed in normal macrophage cell 



4


(u)
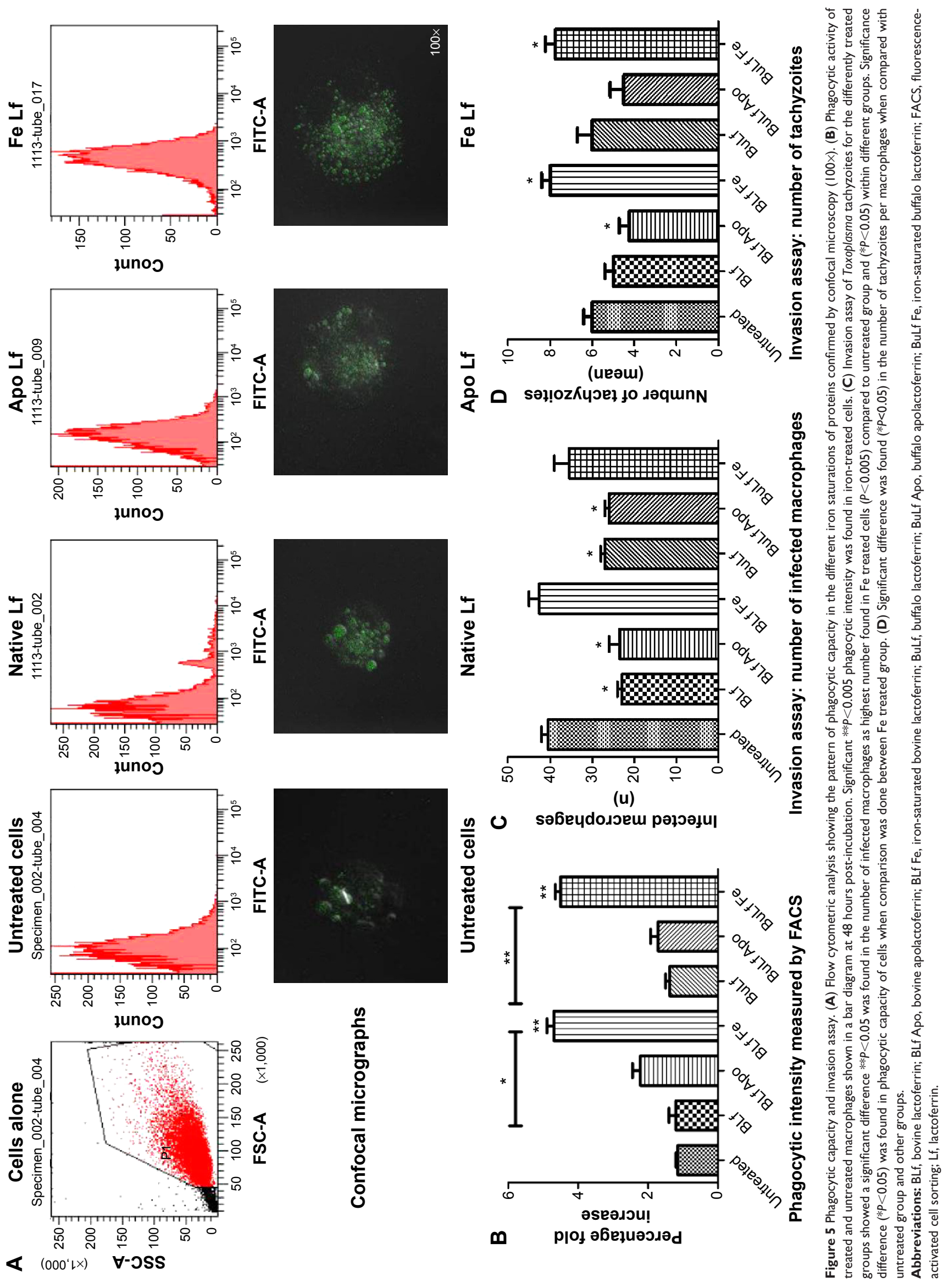

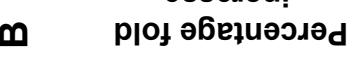

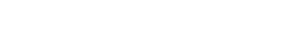






48 hours post-incubation

C

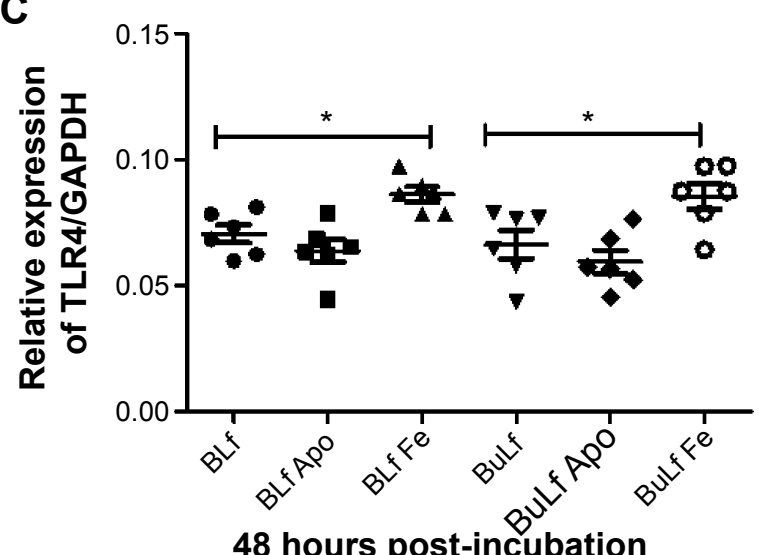

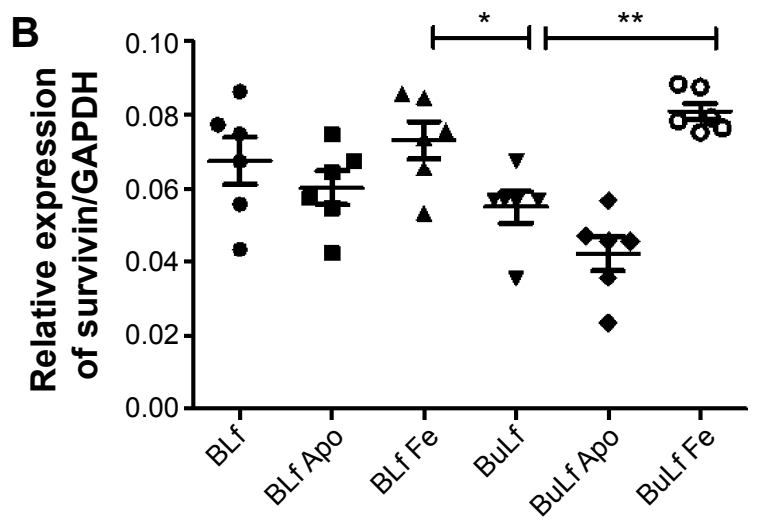

48 hours post-incubation

D

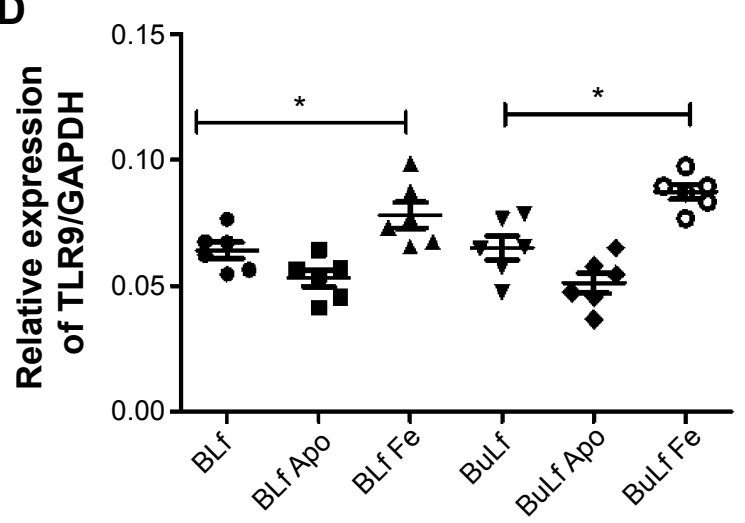

48 hours post-incubation

E

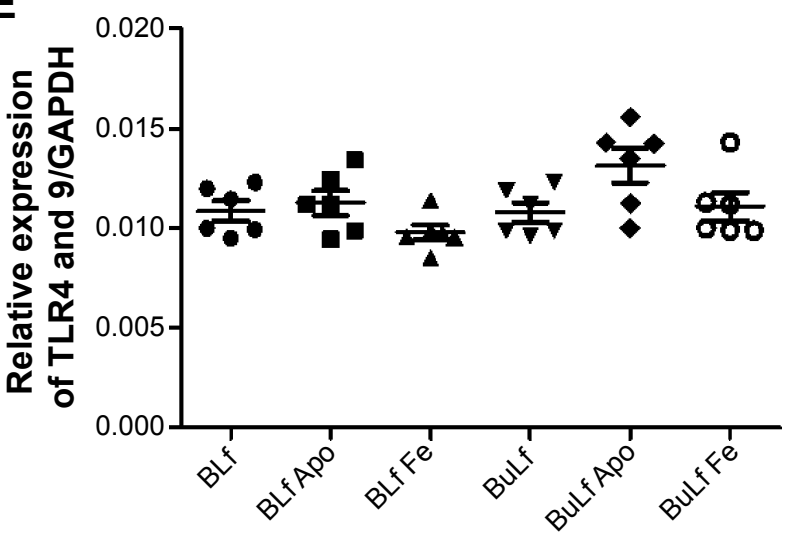

\section{Effect of TLR antagonist}

Figure 6 Expression levels of the different genes. (A) All treated cells showed a significant difference $(P<0.005)$ in MDRI gene regulation (B) Survivin gene expression compared with the untreated group and other groups at 48 hours post-incubation. (C) \& (D) All the treated cells showed elevated levels of TLR4 and 9 expression and significant high levels were observed in Fe-Lf treated cells when compared with untreated group and other groups. (E) The TLR antagonist achieved a 7-9-fold downregulation of expression in the different groups. A significant difference $(P<0.05)$ was found between the iron-saturated lactoferrin-treated group compared with the other groups. $*=P<0.05 ; * *=P<0.005$.

Abbreviations: BLf, bovine lactoferrin; BLf Apo, bovine apolactoferrin; BLf Fe, iron-saturated bovine lactoferrin; BuLf, buffalo lactoferrin; BuLf Apo, buffalo apolactoferrin; BuLf Fe, iron-saturated buffalo lactoferrin; GAPDH, glyceraldehyde-3-phosphate dehydrogenase; TLR, Toll-like receptor.

and no cellular processes will be observed, the proposed mechanism of action has been shown in Figure 7.

\section{Discussion}

Lactoferrin is an iron-binding protein and is present naturally in milk and colostrum in its native form (with one iron molecule). ${ }^{25}$ Lactoferrin can be isolated from many mammals, including humans, cattle, buffalo, mare, camel, goat, horse, and mouse. ${ }^{26,27}$ There is $70 \%$ homology between human lactoferrin and lactoferrin isolated from other species. ${ }^{28}$ Human and bovine lactoferrin proteins have been used extensively in various studies, but buffalo lactoferrin has not been used 
A

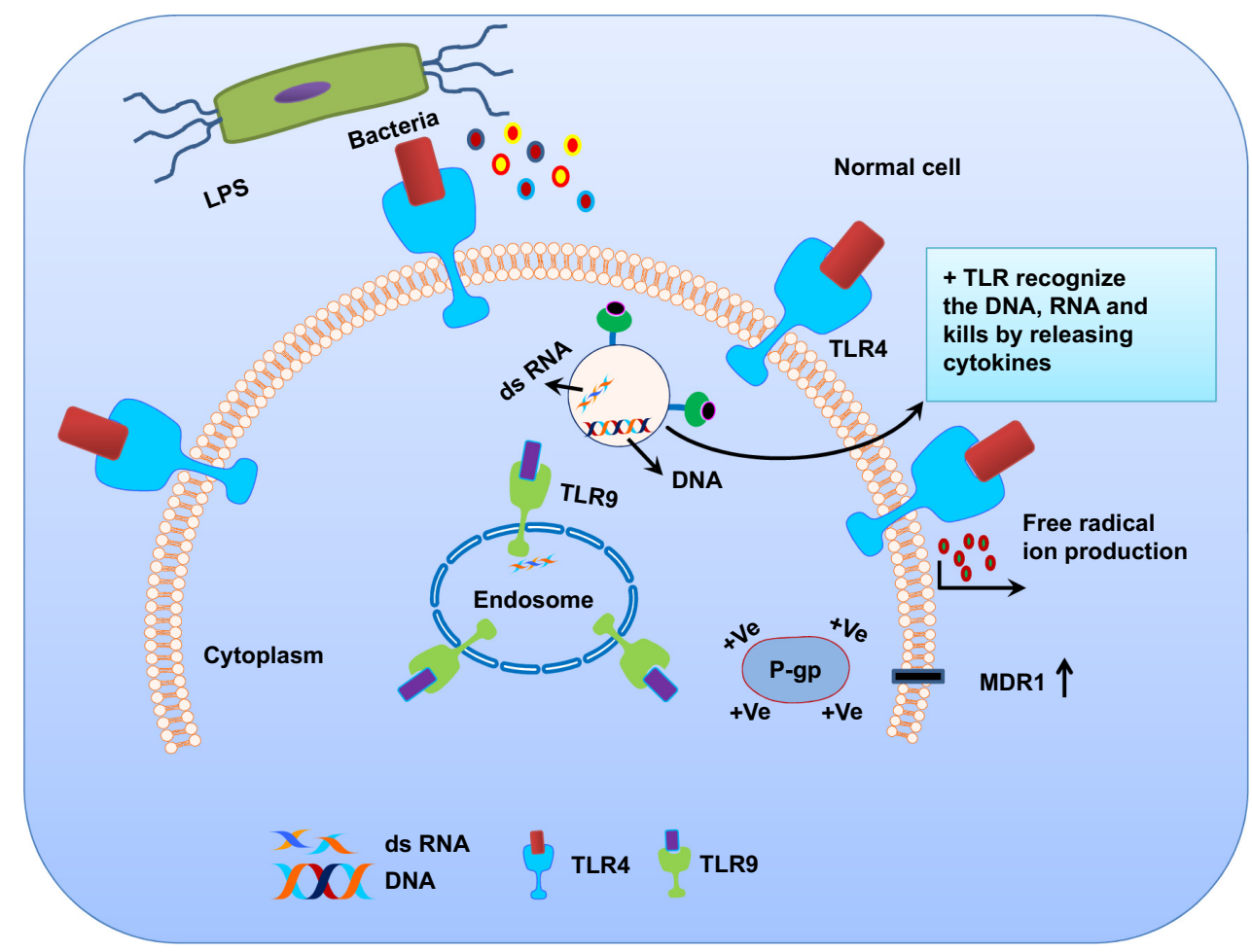

B

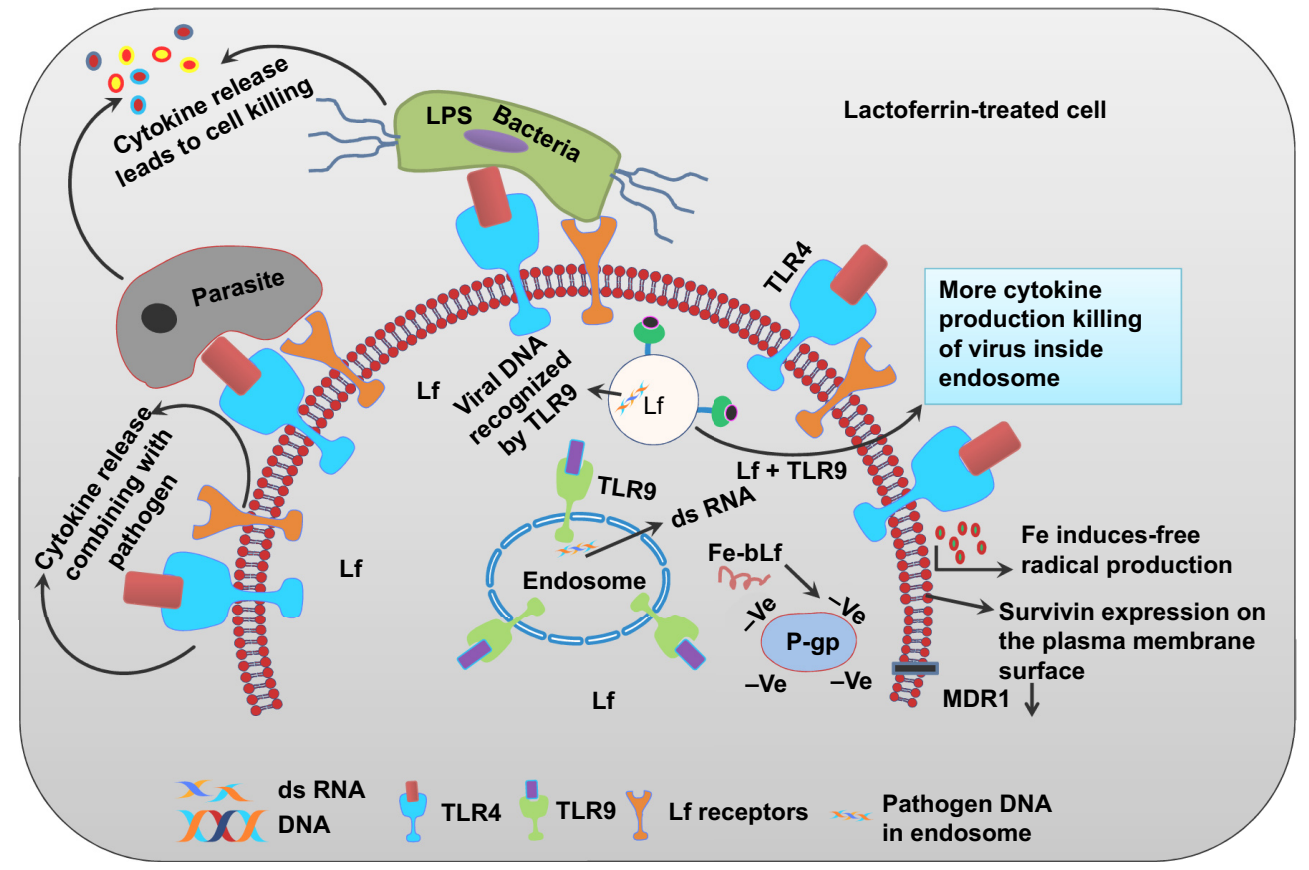

Figure 7 (A) Normal cell showing less TLR expression, less free radical production, and less phagocytic capacity. (B) Lactoferrin-treated cell showing better phagocytosis, more TLR signaling, more free radical production, and less MDRI gene expression after treatment with iron-saturated lactoferrin.

Abbreviations: ds RNA, double-stranded RNA; LPS, lipopolysaccharide; Lf, lactoferrin; P-gp, P-glycoprotein; TLR, Toll-like receptor.

till date. There are some differences in amino acid sequence, glycolysation sites, and the superimposed structure of bovine, buffalo, human, and mouse lactoferrins. ${ }^{28-31}$ These differences can account for the different microbial properties of these proteins. Previous studies have demonstrated the effect of bovine lactoferrin on both macrophage cell lines and tumor cell lines. ${ }^{6,32,33}$ The present study demonstrates the effects of lactoferrin isolated from bovine and buffalo origin with different degrees of iron saturation (ie, the apo, mono, and holo forms) on RBCs and macrophages. To the best of 
our knowledge, the present study is the first to use buffalo lactoferrin along with bovine lactoferrin to analyze the different parameters and undertake a morphometric analysis of RBCs and macrophages. This study can guide us as to how the different origins of lactoferrin can be used to investigate the future aspect on either development of infectious disease or its control.

The lactoferrin protein used in the present study was isolated from bovine and buffalo colostrum using ion exchange chromatography, ${ }^{34}$ and its purity was found to be $90 \%$. Lactoferrins with various degree of iron saturation and at different concentrations were used for dose standardization. RBCs and a leukemia cell line were used to test different forms of lactoferrin because these cells mimic the function of human cells (macrophages, cells of defense system). Among the leukemia cell lines, THP1 is the best one to use when studying any parameter mimicking human function, so this cell line was selected. ${ }^{35}$ Negligible differences were observed in the sizes of RBCs and macrophages after treatment with lactoferrins from both origins.

Production of ROS allows measurement of the respiratory burst, oxidative stress and free radical production which are different phenomenas used in cell biology. Production of ROS inside macrophages indicates the ability of these cells to destroy an intracellular microbe, and oxidative stress means that the cell may die due to high levels of ROS, which are mainly observed in brain cells, as in Alzheimer's disease. Our study was mainly focused on the killing effect generated by macrophages via production of free radical ions. We found that the production of ROS was dose-dependent in all types of treated macrophages. However, iron-saturated lactoferrin-treated cells showed enhanced production of ROS, which can be generated in live cells only and these cells have shown enhanced phagocytic activity. These cells therefore show high activation and more killing activity against intracellular parasite.

Slightly different results for ROS production were found between buffalo lactoferrin and bovine lactoferrin. Previous studies using a lactoferrin concentration of $25-100 \mu \mathrm{M} / \mathrm{mL}$ had confirmed production of ROS in RBCs. ${ }^{36}$ In our study, when RBCs were treated with the various lactoferrins at $40 \mu \mathrm{M} / \mathrm{mL}$ concentration and investigated for ROS production, increased ROS was observed in the iron-saturated lactoferrin group when compared with the untreated groups. ROS in RBCs can be explained on the basis of various metabolic reactions occurring inside these cells. RBCs are known to have many surface receptors for lactoferrin. ${ }^{37}$ Transferrin and lactoferrin may accept an electron while they bind to their co-receptors on the surface of the membrane and convert $\mathrm{Fe}^{3+}$ to $\mathrm{Fe}^{2+}$. Therefore, lactoferrin can act as a final acceptor and initiate oxidative phosphorylation at the plasma membrane.

As we know, glycolysis in RBCs occurs through pentose phosphate pathway, and many reactions during this process may lead to generation of free radical ions. Further, the mono form of lactoferrin is known to take up these ions, leading to reduced ROS production in $\mathrm{RBCs}$. When no iron is available in lactoferrin (the apoferritin form), free metal ions can bind and less ROS production is found. However, when there is no free metal binding site present on the lactoferrin protein (holo form), there is no binding of free metal ions, and these ions ultimately lead to production of ROS on the RBC membrane by lipid phosphorylation. Fenton's reaction ${ }^{38-41}$ is the major reason for ROS production on RBCs, whereby an iron molecule may combine with any free radical, leading to production of $\mathrm{Fe}^{3+}$ and $\mathrm{OH}^{-}$ions that may contribute to production of free radicals. The present study demonstrates that iron-saturated protein, having no free site or free iron molecule, may lead to higher ROS production. ${ }^{36}$

When these proteins were incubated with macrophages, various cellular processes were observed. There was no morphological difference in size of macrophages post-incubation with these proteins. However, there was a 4-5-fold increase level of ROS production in cells incubated with iron-saturated lactoferrin protein compared with the untreated control group. This 4-5-fold increase in production of ROS may be due to the cellular respiratory burst due to more activation of macrophages. This ROS production was confirmed by the increased phagocytic capacity of the macrophages observed through confocal microscopy when compared with other lactoferrins and the untreated groups. Previous studies have also shown increased phagocytic activity in peripheral human macrophages to engulf intracellular microorganisms when incubated with iron-saturated lactoferrin. ${ }^{42,43}$ However, the investigators did not report any effect on the uptake of latex beads, which we found in our study to be increased for ironsaturated lactoferrin.

To confirm phagocytic activity after treatment with the different iron-saturated lactoferrins, we performed an invasion assay using $T$. gondii, a virulent parasite that resides inside macrophages for its survival. Toxoplasma has a life span of 8-10 hours, therefore parasites were kept for only 5 hours to calculate the number of engulfed intracellular parasites (number of events). When the invasion study was performed, few tachyzoites were found inside macrophages treated with apolactoferrin, which may have been due to the low iron content in the cytoplasm as a result of chelation by apolactoferrin. 
On the other hand, macrophages treated with iron-saturated lactoferrins contained more intracellular parasites. This may be due to the nutritional factors provided by the cytoplasm in macrophages, which helped the tachyzoites to invade. Again, the phagocytic properties of iron-saturated lactoferrintreated cells were confirmed, with these cells engulfing more tachyzoites. Previous reports have shown the effect of iron chelation on macrophages from various mechanisms which ultimately lead to reduced phagocytic capacity. ${ }^{44-46}$ Iron has been confirmed to be present in the cytoplasm, and has an important role in phagocytosis.

Upregulation of the survivin gene confirmed the tendency of the treated cells to survive, in a dose-dependent and timedependent manner, when compared with the untreated cells. Survivin was found to be expressed in all the forms of lactoferrin, and was consistent with the MTT viability assay. This clearly highlights the beneficial properties of lactoferrin with regard to drug-resistant microbial pathogen strains.

TLR receptors are known to stimulate many cytokines via activation of the nuclear factor kappa B (NF- $\mathrm{BB})$ complex, which reaches the nucleus and starts the transcription process. This transcription leads to binding of NF- $\kappa B$ with DNA and production of various cytokines, chemokines, free radicals, and various inflammatory factors involved in the host defense system. ${ }^{47}$ Activation of natural host cells with lactoferrin may lead to increased activation of the TLR signaling pathways, given that it is already known from previous studies that lactoferrin is a natural immune system booster. ${ }^{48}$ In the present study, when cells were treated with iron-saturated lactoferrin, they produced more free radical ions, TLR expression, therefore these events may lead to killing of pathogen. Whereas, TLR expression was found to be down regulated in presence of its inhibitor. After binding of various microbial antigens to TLR, may lead to their activation and damage to the pathogen. ${ }^{47}$

The MDR1 gene is known to activate various resistant strains through the P-gp promoter. In the present study, P-gp was found to be expressed in lactoferrin-treated cells, but its expression was decreased when compared with the housekeeping gene, indicating the role of lactoferrin in reducing drug after resistance.

In the present study, we found a correlation between survivin expression, free radical production, a fold increase in phagocytic properties, MDR1, and TLR expression when cells were stimulated in the presence of lactoferrin protein.

From the present study, the proposed mechanism explained here, cells become activated after treatment with iron-saturated lactoferrin when compared with untreated cells, whereby various cellular processes take place both inside and outside the cell. The findings reported here will help us to understand the survival of microorganisms inside host cells, and how iron-saturated lactoferrin, a natural milk protein, helps to inhibit the microbes. The present study was performed to investigate the mechanism of action in relation to various intracellular parasites, such as Plasmodium falciparum, Leishmania donovani, and Toxoplasma gondii. Iron-saturated buffalo lactoferrin was used for the first time, and was shown to have effects on ROS production that were different to those of bovine lactoferrin. Buffalo apolactoferrin was associated with reduced ROS production and also less phagocytic activity when compared with bovine lactoferrin. The iron-saturated forms of both these lactoferrins induced increased ROS production and phagocytic activity, indicating that both proteins shows slightly different action on host cells.

This difference in cell function may indicate further changes in inhibiting the microbes. The present study yielded significant results which can ultimately mimic the human cell function. However, further studies of TLR expression in the signaling pathway and protein expression are needed to clarify the mechanism of interaction with host cells. Human peripheral blood mononuclear cells should be used to study the various mechanisms for better interaction mechanism.

By performing these experiments on host cells, one can determine the mechanism by which lactoferrin destroys intracellular microbes. Lactoferrin has been used as an antiparasitic agent, but its effects have been studied in very few parasitic infections and the detailed mechanism is still not clearly understood. This study has contributed to our understanding of the antiparasitic mechanism of lactoferrin. Further research on the detailed mechanism is necessary to conclude the stronger antiparasitic activity of lactoferrin protein.

\section{Conclusion}

The present study shows the effect of natural multifunctional lactoferrin protein on human host cells. The presence or absence of iron in the protein helps to explain why the host cells show enhanced phagocytosis, TLR expression, and free radical production, which can be beneficial to host cells or harmful to pathogens. The antiparasitic mechanism of lactoferrin is as yet not well understood, but present study has elaborated this mechanism by studying different parameters targeted on parasitic host cells. The pathways involved should be studied further to gain a better understanding of the antiparasitic mechanism of lactoferrin protein. 


\section{Acknowledgments}

The authors would like to thank Bhasker Sriramoju for his assistance with drawings. They also thank the Australia-India Strategic Research Fund for its financial support. The authors also thank the Indian Council of Medical Research, New Delhi (India) for providing the financial support.

\section{Disclosure}

The authors report no conflicts of interest in this work.

\section{References}

1. Metz-Boutigue MH, Jolles J, Mazurier J, et al. Human lactotransferrin: amino acid sequence and structural comparisons with other transferrins. Eur J Biochem. 1984;145(3):659-676.

2. Steijns JM, van Hooijdonk AC. Occurrence, structure, biochemical properties and technological characteristics of lactoferrin. Br J Nutr. 2000;84 Suppl 1:S11-S17.

3. Sorensen M, Sorensen SPL. The proteins in whey. Comptes-Rendus des Travaux du Laboratoire Carlsberg. 1939;23:55-99.

4. Gonzalez-Chavez SA, Arevalo-Gallegos S, Rascon-Cruz Q. Lactoferrin: structure, function and applications. Int J Antimicrob Agents. 2009; 33(4):301.e301-e308.

5. Valenti P, Antonini G. Lactoferrin: an important host defence against microbial and viral attack. Cell Mol Life Sci. 2005;62(22): 2576-2587.

6. Yoo YC, Watanabe R, Koike Y, et al. Apoptosis in human leukemic cells induced by lactoferricin, a bovine milk protein-derived peptide: involvement of reactive oxygen species. Biochem Biophys Res Commun. 1997;237(3):624-628.

7. Kanwar JR, Palmano KP, Sun X, et al. 'Iron-saturated' lactoferrin is a potent natural adjuvant for augmenting cancer chemotherapy. Immunol Cell Biol. 2008;86(3):277-288.

8. Maneva A, Taleva B, Maneva L. Lactoferrin-protector against oxidative stress and regulator of glycolysis in human erythrocytes. Z Naturforsch C. 2003;58(3-4):256-262.

9. Kanwar JR, Mahidhara G, Roy K, et al. Fe-bLf nanoformulation targets survivin to kill colon cancer stem cells and maintains absorption of iron, calcium and zinc. Nanomedicine (Lond). 2015;10(1):35-55.

10. Conneely OM. Antiinflammatory activities of lactoferrin. $J$ Am Coll Nutr. 2001;20 Suppl 5:389S-395S.

11. Puddu P, Valenti P, Gessani S. Immunomodulatory effects of lactoferrin on antigen presenting cells. Biochimie. 2009;91(1):11-18.

12. Zheng Y, Qin Z, Ye Q, et al. Lactoferrin suppresses the EpsteinBarr virus-induced inflammatory response by interfering with pattern recognition of TLR2 and TLR9. Lab Invest. 2014;94(11): $1188-1199$

13. Curran CS, Demick KP, Mansfield JM. Lactoferrin activates macrophages via TLR4-dependent and-independent signaling pathways. Cell Immunol. 2006;242(1):23-30.

14. Magnuson JS, Henry JF, Yip TT, Hutchens TW. Structural homology of human, bovine, and porcine milk lactoferrins: evidence for shared antigenic determinants. Pediatr Res. 1990;28(2):176-181.

15. Lu RR, Xu SY, Wang Z, Yang RJ. Isolation of lactoferrin from bovine colostrum by ultrafiltration coupled with strong cation exchange chromatography on a production scale. J Memb Sci. 2007;297(1): 152-161.

16. Bates GW, Wernicke J. The kinetics and mechanism of iron (III) exchange between chelates and transferrin IV. The reaction of transferrin with iron (III) nitrilotriacetate. J Biol Chem. 1971;246(11):3679-3685.

17. Young D, Hicks JM. Method for the automatic determination of serum iron. J Clin Pathol. 1965;18(1):98-102.

18. Feng M, van der Does L, Bantjes A. Preparation of apolactoferrin with a very low iron saturation. J Dairy Sci. 1995;78(11):2352-2357.
19. Rota C, Chignell CF, Mason RP. Evidence for free radical formation during the oxidation of $2^{\prime}-7^{\prime}$-dichlorofluorescin to the fluorescent dye $2^{\prime}-7^{\prime}$-dichlorofluorescein by horseradish peroxidase: possible implications for oxidative stress measurements. Free Radic Biol Med. 1999;27(7-8):873-881.

20. Klegeris A, Bissonnette CJ, McGeer PL. Modulation of human microglia and THP-1 cell toxicity by cytokines endogenous to the nervous system. Neurobiol Aging. 2005;26(5):673-682.

21. Cheung CH, Chen HH, Cheng LT, Lyu KW, Kanwar JR, Chang JY. Targeting Hsp90 with small molecule inhibitors induces the overexpression of the anti-apoptotic molecule, survivin, in human A549, HONE-1 and HT-29 cancer cells. Mol Cancer. 2010;9:77.

22. Melaine N, Liénard MO, Dorval I, Le Goascogne C, Lejeune H, Jégou B. Multidrug resistance genes and p-glycoprotein in the testis of the rat, mouse, guinea pig, and human. Biol Reprod. 2002;67(6):1699-1707.

23. Yang H, Zhou H, Feng P, et al. Reduced expression of Toll-like receptor 4 inhibits human breast cancer cells proliferation and inflammatory cytokines secretion. J Exp Clin Cancer Res. 2010;29:92.

24. Curran CS, Demick KP, Mansfield JM. Lactoferrin activates macrophages via TLR4-dependent and -independent signaling pathways. Cell Immunol. 2006;242(1):23-30.

25. Brock JH. Lactoferrin in human milk: its role in iron absorption and protection against enteric infection in the newborn infant. Arch Dis Child. 1980;55(6):417-421.

26. Green MR, Pastewka JV. Lactoferrin is a marker for prolactin response in mouse mammary explants. Endocrinology. 1978;103(4):151-103.

27. Kikuchi M, Takao Y, Tokuda N, Ohnami Y, Orino K, Watanabe K. Relationship between seminal plasma lactoferrin and gonadal function in horses. J Vet Med Sci. 2003;65(11):1273-1274.

28. Baker EN, Anderson BF, Baker HM, et al. Three-dimensional structure of lactoferrin in various functional states. Adv Exp Med Biol. 1994;357: $1-12$.

29. Baker EN, Baker HM. Molecular structure, binding properties and dynamics of lactoferrin. Cell Mol Life Sci. 2005;62(22):2531-2539.

30. Baker EN, Baker HM. A structural framework for understanding the multifunctional character of lactoferrin. Biochimie. 2009;91(1): $3-10$.

31. Karthikeyan S, Yadav S, Paramasivam M, Srinivasan A, Singh TP. Structure of buffalo lactoferrin at 3.3 A resolution at $277 \mathrm{~K}$. Acta Crystallogr D Biol Crystallogr. 2000;56 Pt 6:684-689.

32. Yoo YC, Watanabe S, Watanabe R, Hata K, Shimazaki K, Azuma I. Bovine lactoferrin and lactoferricin inhibit tumor metastasis in mice. Adv Exp Med Biol. 1998;443:285-291.

33. Yoo YC, Watanabe S, Watanabe R, Hata K, Shimazaki K, Azuma I. Bovine lactoferrin and lactoferricin, a peptide derived from bovine lactoferrin, inhibit tumor metastasis in mice. Jpn J Cancer Res. 1997; 88(2):184-190.

34. Conesa C, Sanchez L, Perez MD, Calvo M. A calorimetric study of thermal denaturation of recombinant human lactoferrin from rice. J Agric Food Chem. 2007;55(12):4848-4853.

35. Auwerx J. The human leukemia cell line, THP-1: a multifacetted model for the study of monocyte-macrophage differentiation. Experientia. 1991; 47(1):22-29.

36. Maneva A, Taleva B, Maneva L. Lactoferrin-protector against oxidative stress and regulator of glycolysis in human erythrocytes. Z Naturforsch C. 2003;58(3-4):256-262.

37. Suzuki YA, Lonnerdal B. Characterization of mammalian receptors for lactoferrin. Biochem Cell Biol. 2002;80(1):75-80.

38. Krzysztof B. Fenton reaction - controversy concerning the chemistry. Ecological Chemistry and Engineering Science. 16(3):347-358.

39. Thomas C, Mackey MM, Diaz AA, Cox DP. Hydroxyl radical is produced via the Fenton reaction in submitochondrial particles under oxidative stress: implications for diseases associated with iron accumulation. Redox Rep. 2009;143(3):102-108.

40. Luo Y, Henle ES, Linn S. Oxidative damage to DNA constituents by iron-mediated fenton reactions: the deoxycytidine family nucleic acids, protein synthesis, and molecular genetics. J Biol Chem. 1996; 271:21167-21176. 
41. Becker K, Tilley L, Vennerstrom JL, Roberts D, Rogerson S, Ginsburg H. Oxidative stress in malaria parasite-infected erythrocytes: host-parasite interaction. Int J Parasitol. 2004;34(2):163-189.

42. Lima MF, Kierszenbaum F. Lactoferrin effects on phagocytic cell function. I. Increased uptake and killing of an intracellular parasite by murine macrophages and human monocytes. J Immunol. 1985; 134(6):4176-4183.

43. Lima MF, Kierszenbaum F. Lactoferrin effects of phagocytic cell function. II. The presence of iron is required for the lactoferrin molecule to stimulate intracellular killing by macrophages but not to enhance the uptake of particles and microorganisms. J Immunol. 1987; 139(5):1647-1651.

44. Fedorko ME. Loss of iron from mouse peritoneal macrophages in vitro after uptake of (55FE)ferritin and (55FE)erritin rabbit antiferritin complexes. J Cell Biol. 1974;62(3):802-814.
45. Zhang Z, Zhang F, An P, et al. Ferroportin1 deficiency in mouse macrophages impairs iron homeostasis and inflammatory responses. Blood. 2011;118(7):1912-1922.

46. Corhay JL, Weber G, Bury T, Mariz S, Roelandts I, Radermecker MF. Iron content in human alveolar macrophages. Eur Respir J. 1992;5(7): 804-809.

47. Kawai T, Akira S. TLR signaling. Cell Death Differ. 2006;13(5): 816-825.

48. Puddu P, Latorre D, Carollo M, et al. Bovine lactoferrin counteracts Toll-like receptor mediated activation signals in antigen presenting cells. PLoS One. 2011;6(7):e22504.

\section{Publish your work in this journal}

Drug Design, Development and Therapy is an international, peerreviewed open-access journal that spans the spectrum of drug design and development through to clinical applications. Clinical outcomes, patient safety, and programs for the development and effective, safe, and sustained use of medicines are a feature of the journal, which has also been accepted for indexing on PubMed Central. The manuscript management system is completely online and includes a very quick and fair peer-review system, which is all easy to use. Visit http://www.dovepress.com/testimonials.php to read real quotes from published authors.

Submit your manuscript here: http://www.dovepress.com/drug-design-development-and-therapy-journal 